\title{
Classification of Erythemato-squamous diseases using Artificial Neural Network and Genetic algorithm
}

\author{
Pushpalata Pujari, Department of CSIT, Guru Ghasidas Vishwavidyalaya, Bilaspur, C.G, India. \\ Pujari.lata@rediffmail.com
}

Abstract : This paper introduces a hybrid model using artificial neural network (ANN) and genetic algorithm (GA) to develop an efficient classification technique for classification of different categories of Erythemato-squamous diseases. Neural network has been extensively used in many applications like classification, regression, web mining, system identification and pattern recognition. Weight optimization in neural network has been a matter of concern for researchers in the field of soft computing. In this paper the weights of ANN are optimized with GA. The proposed hybrid model is applied on the Erythemato-squamous dataset taken from UCI machine learning repository. The data set contains six different categories: psoriasis, seboreic dermatitis, lichen planus, pityriasis rosea, chronic dermatitis and pityriasis rubra pilaris of Erythemato-squamous diseases. The main aim of this paper is to determine the type of Eryhemato-Squamous disease using the hybrid model. The performance of the hybrid model is evaluated using statistical measures like accuracy, specificity and sensitivity. The accuracy of the proposed model is found to be $99.34 \%$ on test dataset. The experimental result shows the effectiveness of the hybrid model in classification of Erythematosquamous diseases.

Keywords-Soft Computing, GA, ANN, GA-ANN and Hybrid model.

\section{INTRODUCTION}

Hybrid approach is a very important technique to get an optimum solution in soft computing. Hybridization is the mixture of pre existing techniques. GA based ANN (GAANN) is an approach which incorporates Genetics algorithm and Neural network [2] [6]. A Neural network attempts to emulate the processing patterns of the biological brain. It results in a program that can learn by comparing solutions to know problems to sets of data presented to it. Neural network learns from past experience and have well adaptability capacity to circumstances. Very complex non linear relationships can be model by neural network. Neural networks are very suitable for classification phenomenon into predetermined class. It is difficult to a get optimized weight by using ANN only. On the other hand a genetic algorithm is the problem solving methods that use the model of living organisms adapting to their environment. Possible solutions are evaluated. The best choices are made, and then more possible solutions are created by combining the factors involved in those first best choices and choosing again. The process continuous until an optimum solution is reached. Genetics Algorithm is useful for finding the optimal solutions for a number of alternative solutions for that problem. GA can be used to optimize the weight of a neural network which is very difficult to find. In this paper genetic algorithm based artificial neural network (GAANN) hybrid approach is used to determine the weights of a multilayer feed forward Neural Network with back propagation learning. In order to obtain final set of weights of the network ANN uses conventional back propagation networks which are based on gradient descent learning. The proposed network is a back propagation network architecture in which GA makes use of real-coded chromosomes, instead of the conventional binary chromosomes to determine the weight of neural network.

\section{BACKGROUND DETAILS}

Some of the related works done in the field of weight optimization of ANN are as follows.

In [7] Qiuwen Zhang, Cheng Wang presented a model of BP ANN based on GA (called GA-BP ANN). For optimization of interconnecting weights and thresholds of back propagation ANN the authors used GA and obtained a better network with GA based back propagation ANN. The proposed model was used to predict the efficiency, precision and stability of earthquake. In [8] ] Ioan Ileana, Corina Rotar, Arpad Incze focused on several results related to optimization of feed-forward neural networks structure by using genetic algorithm.

In [9] Shandilya P, Abhishek A attempted to model and optimize the complex wire electric discharge machining (WEDM) using soft computing techniques. They integrated ANN and GA to optimize the process parameters. In [10] G.Krishna Mohana Rao et.al developed multiperceptron Neural Network models using Neuro solution packages. Genetics Algorithm concept is used to optimize the weighting factor of the network. With this optimized model 
the authors reduced the maximum error considerably. In [11] T.Manju, K.Priya,R. Chitra applied multi layer feed forward neural network (MLFFNN) that integrates genetic algorithm and back propagation network (BPN) for heart attack prediction. The connection weights of MLFFNN were initialized and optimized by GA. They optimized hidden neurons for improving accuracy. In [12] D. Shanthi, S. Sahoo, N. Saravanan proposed a hybrid model of for prediction of stroke disease. The authors used ANN for prediction and the weights of the ANN is optimized with GA to improve the performance of the ANN. Chromosome with the minimum error from the last generation of the GA algorithm was chosen as the initial weights of ANN to make ANN training with the stroke disease data. A comparative study is carried between the predicted and target output of ANN and Hybrid ANN-GA. It was concluded that applying GA algorithm to initialize the weights of ANN can take its advantage of optimization and overcome the shortcomings of the ANN's slow convergence and stuck in the local minima. In [13] T.T. Chow et.al integrated neural network and genetic algorithm in the system optimal control. Practical integration of ANN with GA in achieving the final goal of minimizing operation cost was suggested by the authors. In [14] the authors proposed a hybrid model using ANN and GA to optimize lead ions removal from aqueous solution and obtained maximum of $98.7 \%$ of lead ions removal.

From literature genetic algorithm based ANN has been successively applied in many applications like prediction of heart attack [11], prediction of stroke disease [12], urban traffic forecasting [15], optimization of grinding parameters [16], attack detection [17], damage identification in CFRP plates [18] and prediction of biogas production [19].

This paper is organized into six sections. Section II discusses the background details. The basic process of ANN application is outlined in section III. Basic of genetics algorithm optimization is outlined in Section IV. In section V GA based ANN technique for optimization of weights is developed. The issues related to simulation study and evaluation of the performance is carried out in section VI followed by conclusion in section VII.

\section{ANN WITH BACK PROPAGATION}

The development process for an ANN with back propagation application has eight steps [1] [3].

Step 1: (Data collection) Collect data to be used for the training and testing of the network

Step 2: (Training and testing data separation) divide available data into training and testing data sets. For a moderately sized data set, $60 \%$ of the data can be randomly selected for training, $40 \%$ for testing.

Step 3: (Network architecture) Select network architecture and a learning method.

Step 4: (Parameter tuning and weight initialization) initialize the network weights and parameters .Often, the initial values are important in determining the effectiveness and length of training.

Step 5: (Transformation of data) As per the requirement of ANN, transforms the input data into suitable type and format.

Step 6: (Training) Conduct training using training samples iteratively by presenting input and desired output to the ANN until a stopping criteria is reached. The ANN computes the outputs and compares the predicted output with the target outputs. Error terms are generated basing on the predicted and target outputs. ANN adjusts the weights of the network basing on the error terms until a suitable set of weights are obtained.

Step 7: (Testing) once the training has been completed, test the network with obtained stable set of weights.

The network is ready to use as a stand-alone system or as part of another software system where new input data will be presented to it and its output will be a recommended decision. The stable weights are the final weights of the ANN.

\section{BASIC OF GENETIC ALGORITHM}

1. Initialization: For the problem generate random population of $\mathrm{n}$ chromosomes as initial solutions for the problem. Choose the size of the chromosome N, the cross over probability $\mathrm{P}_{\mathrm{c}}$ and the mutation probability $\mathrm{P}_{\mathrm{m}} \cdot \mathrm{N}: \mathrm{X}_{1}, \mathrm{X}_{2}, \ldots . . \mathrm{X}_{\mathrm{n}}$

2. Fitness: For each of the chromosomes $\mathrm{X}$ in the population calculate the fitness $f\left(X_{1}\right), f\left(X_{2}\right) \ldots . . f\left(X_{n}\right)$

3. New Population: Generate the new population using following steps repeatedly until the completion of new population.

(a) Selection: From the population select two parent chromosomes based on their fitness. The chances of selection of chromosomes with better fitness are high as compared to other chromosomes.

(b) Cross over: Carry out crossover operation with a crossover probability to form new children (offspring).

(c) Mutation: Carry out mutation operation with a mutation probability to avoid the new offspring to be exactly same as the parents.

(d) Accepting: Choose the new offspring to include them in the new population

4. Replace: Replace the old population with the newly generated population for further iteration of the algorithm.

5. Test: If the stopping criterion is reached, return the best solution in current population.

6. Go to step 2 .

\section{LEARNING ALGORITHM FOR GA-ANN}

In this paper the weights of ANN are optimized by using a hybrid model of GA and ANN. In GA based ANN, GA 
algorithm is used to optimize the weights of the neural network [2]. GA works with a population of individual strings, each string representing the weights of the ANN. Each string is assigned a fitness value by applying the weights to $\mathrm{N}$ number of samples in the training set. The fitness value gives an assessment of how good a solution is. The worst fit individuals are replaced with best fit individual. This yield new individual strings as offspring. From the current generation a new generation is generated by selecting best fit individuals for possible solutions. Progressing in this way, after many generations, owing to mixing an exchange of good characteristics, the entire population inherits the best characteristics and turns out to be fit solutions to the problem. A BPN (Back propagation neural network) with 34-12-6 configuration is assumed in this paper.

The number of weights for the network is 480 . Each weight is a real number and a random digit $d=5$ is used for representing a weight value. The string $S$ representing the length of the chromosome is 2400. Fig. 1 represents sample chromosome, randomly generated made up of 34 genes.

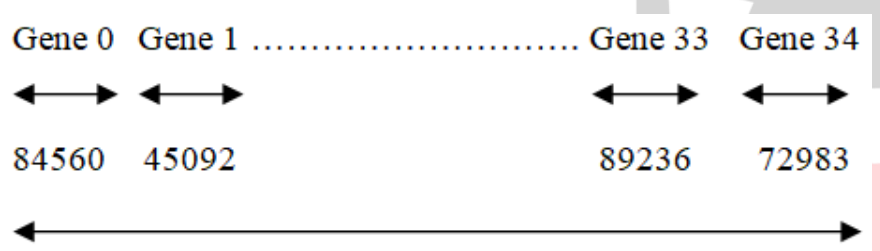

\section{Chromosome}

Fig. 1: Sample Chromosomes randomly generated for the BPN weights

\section{A. Weight Extraction}

To determine the fitness values for each chromosomes, the weight are extracted from each of the chromosomes by using following concept.

Let $\mathrm{x}_{1}, \mathrm{x}_{2}, \ldots, \mathrm{x}_{\mathrm{d} \ldots} \ldots \mathrm{x}_{\mathrm{L}}$ represents a chromosome and $\mathrm{x}_{\mathrm{d}+1}, \mathrm{x}_{\mathrm{d}+2}$, $\ldots . . . \mathrm{x}_{(\mathrm{k}+1) \mathrm{d}}$ represent the $\mathrm{k}^{\text {th }}$ gene $(\mathrm{k}>=0)$ in the chromosome. The weight $\mathrm{w}_{\mathrm{k}}$ is obtained using following equations

$W_{k}=+\frac{\left(X_{k d+2} 10^{d-2}+X_{k d+3} 10^{d-3} \ldots \ldots \ldots \ldots . X_{(k d+1) d}\right)}{10^{d-2}}$

if $5 \leq x_{k d+1} \leq 9$

$W_{k}=-\frac{\left(X_{k d+2} 10^{d-2}+X_{k d+3} 10^{d-3} \ldots \ldots \ldots \ldots X_{(k d+1) d}\right)}{10^{d-2}}$

if $5 \leq x_{k d+2} \leq 9$

B. Algorithm for Fitness function calculation

Algorithm Fitness ( )

\{

Let $\left(\mathrm{Ii}, \mathrm{T}_{\mathrm{i}}\right), \mathrm{i}=1,2 \ldots \mathrm{N}$ where $\mathrm{Ii}=\left(\mathrm{I}_{1 \mathrm{i}}, \mathrm{I}_{2 \mathrm{i}} \ldots \mathrm{I}_{\mathrm{li}}\right)$ and $\mathrm{Ti}=\left(\mathrm{T}_{1 \mathrm{i}}\right.$, $\mathrm{T}_{2 \mathrm{i}} \ldots \mathrm{T}_{\mathrm{ni}}$ ) represent the inputs and desired outputs pairs of the of the network.

For each chromosomes $\mathrm{C}_{\mathrm{i}}, \mathrm{i}=1,2 \ldots \mathrm{P}$ belonging to the current population $\mathrm{P}_{\mathrm{i}}$

\{

Extract weights $\mathrm{W}_{\mathrm{i}}$ from $\mathrm{C}_{\mathrm{i}}$ with the help of equation (1) and (2). Train the back propagation network keeping the weight set $\mathrm{Wi}$ as a fixed for the $\mathrm{N}$ input samples;
Calculate error $E_{i}$ for each of the input instances using the equation

$E_{i}=\sum_{j}\left(T_{j i}-O_{j i}\right)^{2}$

Where $\mathrm{O}_{\mathrm{i}}$ is the output vector calculated by BPN; Find the root mean square $\mathrm{E}$ of the errors $\mathrm{E}_{\mathrm{i}}, \mathrm{i}=1,2 \ldots \mathrm{N}$ by using following equation

i.e.

$E=\sqrt{\sum_{i} \frac{E_{i}}{N}}$

Obtain the fitness value $F_{i}$ of each chromosome in the current population as

$F_{i}=\frac{1}{E}$

\}

Output for each $\mathrm{C}_{\mathrm{i}}, \mathrm{i}=1,2 \ldots \mathrm{p}$;

\}

C.Algorithm for weight optimization

$i \leftarrow 0$;

Initialize the population $\mathrm{P}_{\mathrm{i}}$ with real-coded chromosomes $\mathrm{C}_{\mathrm{ij}}$. Each chromosome initialized represents a set of weight for the back propagation ANN.

While the current population $\mathrm{P}_{\mathrm{i}}$ has not converged \{

Generate fitness values $F_{i j}$ for each $C_{i j}$ for each $C_{i j} \in P_{i}$ using the algorithm Fitness( );

Terminate the individuals with low fitness by replacing them with high fit individuals and make ready the matting pool. Using two point crossover mechanisms reproduce offspring from the parent chromosomes followed by mutation.

Calculate fitness values $\mathrm{F}_{\mathrm{ij}}$ for each $\mathrm{C}_{\mathrm{ij}} \in \mathrm{P}_{\mathrm{i}}$. Combine Parent population and new offspring. Make sorting on total population according to their fitness. Select chromosomes having best fitness for next generation with population size same as initial population

$i \leftarrow i+1$;

\}

Extract weights from $\mathrm{P}_{\mathrm{i}}$ to be used by BPN; \}

END

\section{SIMULATION AND EXPERIMENTAL RESULTS}

The Erythemato-squamous dataset is taken from UCI machine learning repository. Around $60 \%$ samples of the dataset are used for training of the model and the remaining $40 \%$ samples are used for testing the performance of the proposed model.

\section{A. Training of the model}

The training samples are used to train the GA-ANN hybrid model. Architecture of 34-12-6 is used for ANN with BPN. Total number of weights in the network is 480 . 
The input-hidden layer weights and the hidden output layer weights of ANN are extracted using (1) and (2). Each weight is a real number and a random digit $\mathrm{d}=5$ is used for representing a weight value. ANN is trained with initial set of weights. The outputs obtained are compared with the target output and errors are generated. Basing on the errors the weights of ANN are optimized using GA. The string length of chromosome is taken as 2400 . For optimization Initial population $\mathrm{P}_{0}$ of 80 numbers of chromosomes is generated. Using the training data set and weights obtained fitness values are computed for the chromosomes in population $\mathrm{P}_{0}$. The Worst fit chromosomes are replaced with best fit chromosomes and parents are selected randomly. A two point cross over mechanism is used followed by mutation to get new generation. The new generation is combined with old generation and chromosomes for the next generation are evaluated basing on their fitness function. The process is repeated for successive generation with fitness value increasing towards global optimum. Parameters used for GA are number of population $=80$, two point cross over operation with cross over probability $=0.8$, mutation probability $=0.01$, no of generations $=1000$.

\section{B. Performance measures}

Performance of the proposed hybrid model is evaluated by using some very well-known statistical measures classification accuracy, sensitivity and specificity. These measures are defined by true positive (TP), true negative (TN), false positive (FP) and false negative (FN) cases. Fig. 2. represents a matrix showing number of TP, TN, FP, and FN.

\begin{tabular}{c|c|c|c|}
\multicolumn{3}{c}{} & \multicolumn{2}{c}{ Predicted Values } \\
\cline { 2 - 4 } \multicolumn{1}{c|}{ Class } & Positives & Negatives \\
\cline { 2 - 4 } $\begin{array}{c}\text { Actual } \\
\text { Values }\end{array}$ & Positives & $\begin{array}{c}\text { True } \\
\text { Positive(TP) }\end{array}$ & $\begin{array}{c}\text { False } \\
\text { Negative(FN) }\end{array}$ \\
\cline { 2 - 4 } & Negatives & $\begin{array}{c}\text { False } \\
\text { Positive(FP) }\end{array}$ & $\begin{array}{c}\text { True } \\
\text { Negative(TN) }\end{array}$ \\
\cline { 2 - 4 } & &
\end{tabular}

Fig. 2: Matrix for Actual and Predicted cases

Based on the above table following statistical performance measures are evaluated.

Classification accuracy $=$ Total hits $/$ Total Cases

$=(\mathrm{TP}+\mathrm{TN}) /$ Total Cases

Sensitivity $=$ Positive Heats $/$ Total positive Heats

$=\mathrm{TP} /(\mathrm{TP}+\mathrm{FN})$

Specificity $=$ Negative hits $/$ Total Negatives $=\mathrm{TN} /(\mathrm{TN}+\mathrm{FP})$

\section{Experimental Results}

The performance of the model is obtained using (6), (7) and (8). Table 1 and Table 2 show the confusion matrix obtained for training and testing subsets respectively. Table 3 and Table 4 show the performance of the model in terms of sensitivity, specificity and accuracy for training and testing subsets respectively. Table 5 and Table 6 shows number of cases correctly and wrongly classified with classification percentage for training and testing subsets respectively. An accuracy of 99.53 and $99.34 \%$ is obtained by the proposed model with training and testing subsets respectively.

Table 1. Confusion matrix obtained from training subsets

\begin{tabular}{|l|c|c|c|c|c|c|}
\hline Class & Psoriasis & Seboreic dermatitis & Lichen planus & Pityriasisrosea & Chronic dermatitis & Pityriasisrubrapilaris \\
\hline Psoriasis & 66 & 0 & 0 & 0 & 0 & 0 \\
\hline Seboreic dermatitis & 0 & 31 & 0 & 1 & 0 & 0 \\
\hline Lichen planus & 0 & 0 & 0 & 0 & 0 & 0 \\
\hline Pityriasisrosea & 0 & 0 & 0 & 37 & 0 & 0 \\
\hline Chronic dermatitis & 0 & 0 & 0 & 0 & 28 & 0 \\
\hline Pityriasisrubrapilaris & 0 & 0 & 0 & 0 & 0 & 18 \\
\hline
\end{tabular}

Table 2. Confusion matrix obtained from testing subsets

\begin{tabular}{|l|c|c|c|c|c|c|}
\hline Class & Psoriasis & Seboreic dermatitis & Lichen planus & Pityriasisrosea & Chronic dermatitis & Pityriasisrubrapilaris \\
\hline Psoriasis & 46 & 0 & 0 & 0 & 0 & 0 \\
\hline Seboreic dermatitis & 0 & 28 & 0 & 0 & 0 & 0 \\
\hline Lichen planus & 0 & 0 & 35 & 0 & 0 & 0 \\
\hline Pityriasisrosea & 0 & 1 & 0 & 16 & 0 & 0 \\
\hline Chronic dermatitis & 0 & 0 & 0 & 0 & 24 & 0 \\
\hline Pityriasisrubrapilaris & 0 & 0 & 0 & 0 & 0 & \\
\hline
\end{tabular}

Table 3. Performance measures of traing subset

\begin{tabular}{|l|l|l|l|}
\hline Class of Disease & Sensitivity(\%) & Specificity(\%) & Accuracy(\%) \\
\hline Psoriasis & $100 \%$ & $100 \%$ & $100 \%$ \\
\hline Seboreic dermatitis & $96.87 \%$ & $100 \%$ & $99.53 \%$ \\
\hline Lichen planus & $100 \%$ & $100 \%$ & $100 \%$ \\
\hline Pityriasisrosea & $100 \%$ & $100 \%$ & $100 \%$ \\
\hline Chronic dermatitis & $100 \%$ & $100 \%$ & $100 \%$ \\
\hline Pityriasisrubrapilaris & $100 \%$ & $100 \%$ & $100 \%$ \\
\hline
\end{tabular}

Table 4. Performance measures of testing subset

\begin{tabular}{|l|l|l|l|}
\hline \multicolumn{1}{|c|}{ Class of Disease } & Sensitivity (\%) & Specificity(\%) & Accuracy(\%) \\
\hline Psoriasis & $100 \%$ & $100 \%$ & $100 \%$ \\
\hline Seboreic dermatitis & $100 \%$ & $100 \%$ & $100 \%$ \\
\hline Lichen planus & $100 \%$ & $100 \%$ & $100 \%$ \\
\hline Pityriasisrosea & $94.11 \%$ & $100 \%$ & $99.34 \%$ \\
\hline Chronic dermatitis & $100 \%$ & $100 \%$ & $100 \%$ \\
\hline Pityriasisrubrapilaris & $100 \%$ & $100 \%$ & $100 \%$ \\
\hline
\end{tabular}


Table 5: Total Classification Accuracy of Training subset

\begin{tabular}{|l|c|c|}
\hline Cases & $\begin{array}{c}\text { Number of } \\
\text { instances }\end{array}$ & \% of classification \\
\hline Correct & 212 & $99.53 \%$ \\
\hline Wrong & 1 & $0.47 \%$ \\
\hline Total Cases & 213 & \\
\hline
\end{tabular}

Table 6: Total Classification Accuracy of Testing subset

\begin{tabular}{|l|c|c|}
\hline Cases & $\begin{array}{c}\text { Number of } \\
\text { instances }\end{array}$ & \% of classification \\
\hline Correct & 151 & $99.34 \%$ \\
\hline Wrong & 1 & $0.66 \%$ \\
\hline Total Cases & 152 & \\
\hline
\end{tabular}

\section{CONCLUSION}

This paper proposes a hybrid model for weight optimization of ANN using GA optimization based learning rule for classification of Erythemato-squamous diseases. The proposed model is applied on Erythemato-squamous dataset taken from UCI machine learning repository. The weights of ANN are optimized with GA. Performance of the hybrid model is evaluated in terms of sensitivity, specificity and accuracy..The simulation result shows the effectiveness of the hybrid model with classification accuracy of $99.34 \%$ on test samples. The proposed model can be effectively used for the diagnosis of different types of Eryhemato-Squamous Diseases.

\section{REFERENCES}

[1] Hagan Demuth Beale, “ Neural Network Design”, 1996 Cengage Learning, India PVT Ltd. ISBN-13:978-81-315-0395-9, ISBN10:81-315-0395-X

[2] S.Rajasekaran, G.A.Vijayalakshmi Pai, "Neural Networks, Fuzzy Logic, and Genetic Algorithms Synthesis and Applications", 2003 PHI publication, ISBN-81-203-2186-3

[3] Simon Haykin, "Neural Networks A Comprehensive Foundation", 1999, Pearson Prentice Hall, ISBN 81-77-58-8524

[4] Majhi.R, Majhi.B, Mishra .M, Panda G, Panigrahi S.K, "Forecasting of Retail Sales Data Using Differential Evolution", 2009 World Congress on Nature \& Biologically Inspired Computing( NaBIC 2009).

[5] Majhi.R, Majhi.B, Rout M, Mishra S,, Panda G, "Efficient Sales Forecasting Using PSO Based Adaptive ARMA Model”, 2009 World Congress on Nature \& Biologically Inspired Computing (NaBIC 2009).

[6] Ms.Dharmistha D.Vishwakarma, "Genetic Algorithm based Weights Optimization of Artificial Neural Network" , International Journal of Advanced Research in Electrical, Electronics and Instrumentation Engineering Vol. 1, Issue 3, PP: 206-211,August 2012, ISSN 2278 -8875

[7] Qiuwen Zhang, Cheng Wang, “ Using Genetic Algorithm to Optimize Artificial Neural network: A Case study on Earthquake Prediction", WGEC '08 Proceedings of the 2008 Second International Conference on Genetic and Evolutionary Computing, pp. 128-131, IEEE Computer Scoiety Washington, DC, USA USA (02008, ISBN: 978-0-7695-3334-6
[8] Ioan Ileana, Corina Rotar, Arpad Incze , "The Optimization of Feed Forward Neural Networks Structure using Genetic Algorithms" , Proceedings of the International Conference on Theory and Applications of Mathematics and Informatics ICTAMI 2004, Thessaloniki, Greece 223

[9] Shandilya P, Abhishek A, "Artificial Neural Network Modeling and Optimization using Genetic Algorithm of Machining Process ", Journal of Automation and Control Engineering Vol. 2, No. 4, pp: 348-352, December 2014

[10] G. Krishana Mohana Rao, G. Ranga Janardhana, D. Hanumantha Rao, M. Shrinivas Rao,“ Development of Hybrid Model and Optimization of Metal Removal Rate in Electric Discharging Machining using Artificial Neural Network and Genetics Algorithm", ARPN Journal of Engineering and Applied Sciences , Vol.3, No.2, February 2008, pp: 19-30, ISSN: 1819-6608

[11] T. Manju, K. Priya, R. Chitra, "Heart Disease Prediction System using Weight Optimized Neural Network", International Journal of Computer Science and Management Research Vol. 2 Issue 5, pp: 2391-2397 May 2013, ISSN 2278-733X

[12] D. Shanthi, S. Sahoo, N. Saravanan ,’Evolving Connection Weights of Artificial Neural Network Using Genetic Algorithm with Application to the Prediction of Stroke Disease", International Journal of Soft Computing Year:2009, Vol. 4, Issue:2 $, \mathrm{pp}:$ 95-102.

[13] T.T. Chow, Z. Lin and C.L. Song, G.Q. Zhang, "Applying Neural Network and Genetics Algorithm in Chiller System Optimization", Seventh International IBPSA Conference Rio de Janeiro, Brazil, pp:1059-1069,August 13-15, 2001.

[14] Yamin Yasin, Faujan Bin H. Ahmad, Mansour GhaffariMoghaddam, Mostafa Khajeh, "Application of a hybrid artificial neural network-genetic algorithm approach to optimize the lead ions removal from aqueous solutions using intercalated tartrate- $\mathrm{Mg}-\mathrm{Al}$ layered double hydroxides", Environmental Nanotechnology, Monitoring \& Management, Volumes 1-2, November 2014, Pages 2-7.

[15] Asif Raza and MingZhong, "Lane-based short-term urban traffic forecasting with GA designed ANN and LWR models", Transportation Research Procedia, Volume 25, 2017, Pages 1430-14.

[16] Vipin Gopan, K. Leo Dev Wins and Arun Surendran, "Integrated ANN-GA Approach For Predictive Modeling And Optimization of Grinding Parameters With Surface Roughness As The Response", Materialstoday proceedings, Volume 5, Issue 5, Part 2, 2018, Pages 12133-12141.

[17] Soodeh Hosseini and Behnam Mohammad HasaniZade," New hybrid method for attack detection using combination of evolutionary algorithms, SVM, and ANN", Computer Networks, Volume 173, 22 May 2020, 107168

[18] Guliherme Ferreira Gomes, Fabricio Alves de Almeida, Diego Morais Jungueira, Sebastiao Simoes da Cunha Jr, Antonio Carlos Ancelotti Jr., "Optimized damage identification in CFRP plates by reduced mode shapes and GA-ANN methods", Engineering Structures, Volume 181, 15 February 2019, Pages 111-123.

[19] Tanja Beltramo, Mochael Klocke and Bernd Hitzmann, "Prediction of the biogas production using GA and ACO input features selection method for ANN model", Information Processing in Agriculture, Volume 6, Issue 3, September 2019, Pages 349-356 